\title{
Motion Interpolation with Bennett Biarcs
}

\author{
Hans-Peter Schröcker and Bert Jüttler
}

\begin{abstract}
We present an interpolation scheme for first order Hermite motion data (two positions with associated instantaneous screws specifying the tangent vector fields) that is based on a generalization of the classic biarc construction to curves on quadrics. The result is a sequence of Bennett motions. These motions possess several properties that make them particularly useful for motion interpolation, especially for applications requiring collision detection. We suggest methods for choosing the free parameter that determines the interpolating pair of Bennett motions and we demonstrate how to obtain an interpolation algorithm which is invariant with respect to changes in the moving and the fixed coordinate frame.
\end{abstract}

\section{Introduction}

Bennett motions are named after G. T. Bennett who introduced them as coupler motions of movable closed spatial chains with four revolute joints [2,3]. Since then, Bennett's mechanism and the associated coupler motion have continuously attracted the attention of many researchers. See [6] for an overview with many related references.

Bennett motions possess several properties that make them interesting for motion interpolation, in particular for collision detection:

- They are rational motions ${ }^{1}$ of degree four (i.e., all point trajectories are rational quartic curves),

Hans-Peter Schröcker

Unit Geometry and CAD, University Innsbruck, e-mail: hans-peter.schroecker@uibk.ac.at

Bert Jüttler

Institute of Applied Geometry, Johannes Kepler University Linz, e-mail: bert.juettler@jku.at

${ }^{1}$ See the surveys $[10,14]$ for more information on the theory and applications of piecewise rational motions, in particular on interpolation algorithms. 
- the motion synthesis is straightforward (see [6]),

- the inverse motion, obtained by interchanging the rolses fixed and moving frame, is again a Bennett motion, as one can conclude from the mechanical realization,

- their images under Study's kinematic mapping are conic sections, hence the Study parameters of Bennett motions can be chosen as rational quadratic functions, and

- the curves on the Plücker quadric which are generated by moving lines possess a particularly low degree - they are simply quadratic.

We present a method for Hermite motion interpolation by Bennett motions. To given start position $a$ and end position $b$ along with corresponding instantaneous screws (velocity vector fields) $A$ and $B$, we construct a rational, velocity continuous motion that is composed of two Bennett motions. However, it is required that the two instantaneous screws possess the same chirality.

Our interpolation scheme is based on the well-known generalization of the planar biarc construction to biarcs on quadrics. This generalization has first been investigated by Wang and Joe in [16]. It has later been used in various problems of applied geometry $[7,12,15]$.

\section{Biarcs}

A biarc in the plane is a pair of circular arcs, joined with tangent continuity, that connects a pair $(a, A)$ of start point $a$ and oriented start tangent $A$ with a pair $(b, B)$ of end point $b$ and oriented end tangent $B$. The concept of biarcs has been extended to biarcs on quadrics [16]. A biarc on a quadric $Q$ is a pair of tangent continuous conic arcs, contained in the quadric $Q$, that connects a pair $(a, A)$ of quadric point $a$ and quadric tangent $A$ with a pair $(b, B)$ of quadric point $b$ and quadric tangent $B$.

For given data in space, the quadric $Q$ can always be chosen as the unique sphere which is determined by the two pairs of point and tangents. Its center is the intersection of the bisector plane of the two points with the two normal planes. This choice leads to the special case of biarcs in space consisting of two circular arcs [7].
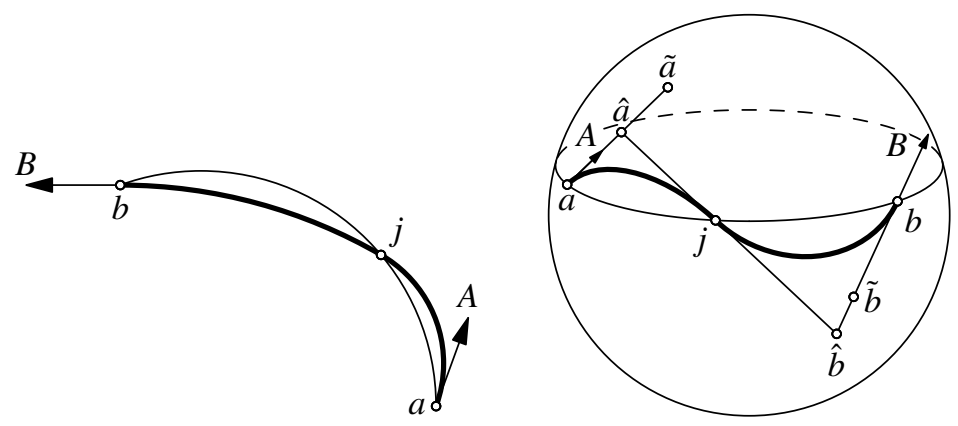

Fig. 1 Biarcs in the plane and on a sphere 
We are interested in the case where the quadric $Q$ is also given. Let us recall the fundamental properties of biarcs on quadrics (cf. [13, pp. 292-296] or [16]). The boundary data $(a, A),(b, B)$ span a three-dimensional space in which the biarc construction takes place. Therefore, it is sufficient to consider only quadrics in a space of three dimensions.

The existence of a biarc on a given quadric cannot be guaranteed in general. It exists if and only if ${ }^{2}$ both quadric tangents $A$ and $B$ lie on the same side of $Q$, that is, if $F$ is an algebraic equation of degree two with zero set $Q$, we have $F(x) F(y) \geq 0$ for all points $x \in A$ and $y \in B$. This condition is always fulfilled if the quadric $Q$ (or at least its intersection with the three-space spanned by the biarc data) is oval. The existence of a single biarc on a quadric to given data implies the existence of a one-parameter family of quadric biarcs. The locus of possible joint points is a conic $J \subset Q$ through $a$ and $b$.

\section{Bennett biarcs}

Recall that Study's kinematic mapping, which provides a quadric point model for Euclidean displacements, associates with every Euclidean displacement $\gamma$ a unique point $c$ on the Study quadric $S \subset P^{7}$. Conversely, every point of $S$ (except for the points of a certain three-dimensional generator space) corresponds to a Euclidean displacement.

The eight entries of the homogeneous coordinate vector of $c=\left[c^{\prime}, c^{\prime \prime}\right]^{T}$ are called the Study parameters of $\gamma$. They satisfy the relation $c^{\prime T} \cdot c^{\prime \prime}=0$ and correspond directly to the description of Euclidean displacements via dual quaternions $c^{\prime}+\varepsilon c^{\prime \prime}$, see $[4$, Chapter $13, \S 8]$ or $[11$, Section 4.5]. In the sequel we view $c$ either as point in $P^{7}$, as homogeneous coordinate vector of dimension eight or as dual quaternion. See [8] for more information on Study's kinematic mapping, including formulas for conversion between Study parameters and homogeneous $4 \times 4$ transformation matrices representing displacements.

The kinematic image of a one-parameter motion is a curve on the Study quadric $S$. Straight lines correspond either to continuous rotations about a fixed axis or to translations in a fixed direction. Conic sections on $S$ were recently recognized as kinematic images of the coupler motion of Bennett mechanisms [5, 6] (including degenerate cases caused by conic points in the three-space $\left.c^{\prime}=[0,0,0,0]^{T}\right)$. Hence, a biarc on the Study quadric $S$ is the concatenation of two Bennett motions, which we will refer to as a Bennett biarc.

According to [1] the instantaneous screw of a Bennett motion is always regular (the pitch is never zero or infinity). This also follows from the fact that all tangents of the kinematic image conic lie on the same side of $S$. As a consequence, the instantaneous screws of a Bennett motion always have the same chirality.

\footnotetext{
${ }^{2}$ Note that [16] considers quadrics in affine spaces. Therefore additional constraints on the biarc data are needed in order to ensure finiteness of the two conic arcs. For our purpose this is not necessary.
} 
The kinematic images of Bennett motions are conic sections on the Study quadric. The tangents of the Study quadric correspond to instantaneous screws and screws of different chirality lie on different sides of the Study quadric. Translating the well-known facts about biarcs on quadrics into the language of kinematics, we obtain the following result.

Theorem 1. Two pairs $(a, A),(b, B)$ of position and instantaneous screw (velocity vector field) can be interpolated by a Bennett biarc if and only if $A$ and $B$ are of the same chirality. In this case there exists a one-parameter family of Bennett biarcs. All possible joint positions form another Bennett motion.

Every biarc has a single degree of freedom. On the one hand, a particular solution can be identified via optimization of a suitable objective function. On the other hand, the free parameter can also be used to generate bi-invariant interpolation schemes, as described below.

\section{Interpolation by Bennett biarcs}

The computation of Bennett biarcs follows the procedures described in [13, pp. 292296] or [16]. We give an outline of the basic steps. Consider a pair $(a, A)$ of start position $a$ and initial instantaneous screw $A$ and a pair $(b, B)$ of end position $b$ and final instantaneous screw $B$. We view $a$ and $b$ as points on the Study quadric $S \subset P^{7}$; $A$ and $B$ are tangents of $S$ in $a$ and $b$, respectively. Denote by $\langle x, y\rangle=x_{0} y_{4}+x_{1} y_{5}+$ $x_{2} y_{6}+x_{3} y_{7}$ the bilinear form associated to the Study quadric and consider two points $\tilde{a} \in A, \tilde{b} \in B$ such that $a \neq \tilde{a}$ and $b \neq \tilde{b}$ (Figure 1). The span of $\hat{a}=a+\lambda \tilde{a}$ and $\hat{b}=b+\mu \tilde{b}$ is tangent to $S$, if

$$
\langle a, b\rangle+\lambda\langle\tilde{a}, b\rangle+\mu\langle a, \tilde{b}\rangle+\lambda \mu(\langle\tilde{a}, \tilde{b}\rangle \pm \varphi)=0, \quad \varphi^{2}=\langle\tilde{a}, \tilde{a}\rangle \cdot\langle\tilde{b}, \tilde{b}\rangle .
$$

Solving for $\mu$ and computing the point of tangency $j=j(\lambda)$ we obtain a rational quadratic parametrization of the joint conic in terms of $\lambda$. The sought conic arcs can be parametrized as rational Bézier curves on $S$ with control points $a, \hat{a}, j$ and $j, \hat{b}, b$ and suitably chosen weights, respectively. If desired, the curves may be reparametrized in order to ensure $C^{1}$-continuity (and not just geometric continuity). The sign of $\varphi$ in (1) has to be chosen such that the given orientation of $A$ and $B$ is matched. In case of $\varphi^{2}<0$ no real solution exists.

The given Hermite motion data $(a, A),(b, B)$ with instantaneous screws $A, B$ of the same chirality admits a one-parameter family of interpolating Bennett biarcs. The choice of the joint position $j$ is the only degree of freedom. It may be left to the motion designer, but it can be automatized as well. We propose two strategies for choosing a proper joint position. Both of them yield a bi-invariant interpolation scheme and can be viewed as generalizations of the "equal chord biarcs", which are well-known from planar geometry.

Denote the rotation angles of the relative displacements $a \rightarrow j$ and $j \rightarrow b$ by $\varphi_{a}$ and $\varphi_{b}$, respectively, and the translation distances by $\eta_{a}$, and $\eta_{b}$, respectively. We 
propose to choose the joint point $j$ so that either $\varphi_{a}=\varphi_{b}$ (equal angle biarc) or $\eta_{a}=\eta_{b}$ (equal distance biarc). The geometric significance of the choices of the joint point guarantees bi-invariance of the interpolation scheme.

Theorem 2. On every connecting joint conic arc of $a$ and $b$ there exists exactly one joint position that yields an equal angle biarc and exactly one joint position that yields an equal distance biarc.

Proof. By the intermediate value theorem, there exist at least two solutions, one on every connecting conic arc between $a$ and $b$. From [17, p. 12] it follows that the parameter value $t$ for equal angle biarcs is a root of the quadratic equation

$$
\left(\sum_{i=0}^{3} b_{i}^{2}\right)^{1 / 2} \sum_{i=0}^{3} a_{i} j_{i}(t)-\left(\sum_{i=0}^{3} a_{i}^{2}\right)^{1 / 2} \sum_{i=0}^{3} b_{i} j_{i}(t)=0 .
$$

Hence, the solutions are unique. The half rotation angle $\varphi$ and half translation distance $\eta$ of a displacement $c=\left[c_{0}, \ldots, c_{7}\right]^{T}$ can be computed by the formulas

$$
\cot \varphi=-c_{0}\left(c_{1}^{2}+c_{2}^{2}+c_{3}^{2}\right)^{-1 / 2}, \quad \eta=c_{4}\left(c_{1}^{2}+c_{2}^{2}+c_{3}^{2}\right)^{-1 / 2}
$$

[17, p. 115]. Hence, the equal distance biarc to the given data corresponds to the equal angle biarc to the data obtained by swapping the first and fifth coordinate and multiplying them by -1 .

Equation (3) demonstrates that there is no essential difference in the computation of equal angle and equal distance biarcs. For the actual computation it is, however, better to use (2) since it is quadratic while (3) amounts to solving a quartic equation. An example for a motion generated by Bennett biarc interpolation is available http://geometrie.uibk.ac.at/institutsangehoerige/schroecker/dld/bennett-biarcs/.

Note that Bennett biarcs exist only if the boundary screw motions possess the same chirality. In order to handle boundary data with screws of different chirality or even singular screws, one may either use a segment of a general rational motion (cf. [10]) or one can interpolate solely the position data with a Bennett motion. Clearly, the latter approach produces velocity discontinuities, which can, however, be expected to be small, since the motion should be relatively close to a motion with singular instantaneous screws (translations or rotations).

\section{Collision detection between moving and fixed polyhedra}

The low algebraic degree of Bennett motions, the equally simple inverse motion and especially the parametrization in Study parameters make certain problems of collision detection particularly simple. We consider a moving polyhedron, which is subject to a Bennett motion, in an environment of static polyhedra. All polyhedra are assumed to be triangulated, and thus the elementary collision predicate is a test for interference of a moving and a static triangle. 
In Computer Animation it is common to use affine motions with linearly parametrized trajectories, which are obtained by linear keyframe interpolation. In this case, the test for interference between a fixed and a moving triangle requires solely the analysis of polynomials of degree less or equal than three, cf. for example [9]. Indeed, it suffices to test for planarity of all $\left(\begin{array}{l}6 \\ 4\end{array}\right)=15$ quadruples generated by the six vertices of points, and at most three of them are moving (with linearly parametrized trajectories).

In the case of Bennett motions, which preserve the rigidity of the moving object, the situation is only slightly more complicated: Now 15 quartic polynomials need to be analyzed. More precisely, three different types of contact between two triangles are possible:

- Moving point vs. fixed plane: The trajectories of points are rational quartics of degree four. Substitution into the equation of the plane gives a quartic polynomial.

- Moving plane vs. fixed point. Equivalently one may consider the inverse motion, i.e., the situation in the moving coordinate system. There, a moving point has to be tested with a fixed plane. Since the inverse motion is a Bennett motion too, whose Study parameters are the conjugates of the dual quaternions, this case can be dealt with as before.

- Moving line vs. fixed line: In order to describe the trajectory of a straight line, we observe that the transformed Plücker coordinates are quadratic in the Study parameters and hence rational of degree four, see [17, p. 109] or [4, Chapter 13, $\S 8]$. Since the intersection condition for straight lines is bilinear in the line's Plücker coordinates, the intersection test between a moving and a fixed line also leads to a quartic polynomial.

Clearly, similar to the case of linear keyframe interpolation, the three intersection tests represent necessary conditions for collisions between the fixed and the moving triangle. If they are satisfied, one has to compute the intersection points and to check whether or not they are contained in the two triangles.

We illustrate the proposed interpolation procedure and collision tests with an example. We want to interpolate two pairs $(a, A),(b, B)$ of Hermite motion data; $a$ and $b$ are points of the Study quadric $S, A=a \vee \tilde{a}$ and $B=b \vee \tilde{b}$ are tangent to $S$ :

$$
\begin{gathered}
a=[1,0,0,0,0,0,0,0]^{T}, \quad \tilde{a}=[0,0,0,1,0,0,0,-1]^{T}, \\
b=[10,10,0,0,5,-5,-21,-19]^{T}, \quad \tilde{b}=[-20,0,0,0,5,5,30,8]^{T} .
\end{gathered}
$$

The corresponding start and end position ( $a$ and $b$ ) are visualized in Figure 2. The instantaneous screws are indicated by a few transparent end effector positions (which is represented by a triangle) and labeled by $A$ and $B$, respectively. A rational parametrization of the joint conic $J$ is

$$
\begin{aligned}
J: j(t)= & t^{2}[-100,280,0,-190,235,-45,-18,-190]^{T}+ \\
& t[-80,100,0,100,0,-100,-510,-370]^{T}+[200,0,0,0,0,0,0,0]^{T} .
\end{aligned}
$$


The joint points to the two equal angle biarcs are found by solving the quadratic equation $31 t^{2}+31(\sqrt{2}-1) t+10(-14 \sqrt{2}+19)=0$. The solution $t_{j}=0.3412$ is the one which complies with the given direction of the start and end screw. The corresponding biarc motion is visualized in Figure 2, left. The joint position $j$ is drawn in thick line-style.

Now we perform some collision tests with the equal angle Bennett motion. We consider the moving triangle $P=p_{0} p_{1} p_{2}$ and the fixed triangle $Q=q_{0} q_{1} q_{2}$ where

$$
\begin{aligned}
p_{0} & =[1,1,0,0]^{T}, & p_{1}=[1,0,1,0]^{T}, & p_{2}=[1,0,0,1]^{T}, \\
q_{0} & =[1,2,1,5], & q_{1}=[1,0,2,3], & q_{2}=[1,0,1,5],
\end{aligned}
$$

and restrict ourselves to the second Bennett arc (there is no relevant interference during the first part of the motion) whose parametrization in Study parameters is

$$
\begin{aligned}
J_{2}: j_{2}(t)= & t^{2}[1,0.1711,0,0.1377,0.0805,-0.0906,-0.3896,-0.4711]^{T}+ \\
t \quad[-2.7367,-0.8231,0,-0.2759,-0.3375,0.4856,2.1727,1.9582]^{T}+ & {[1.8516,0.7670,0,0.1380,0.3145,-0.4525,-2.0245,-1.7056]^{T} . }
\end{aligned}
$$

Moreover, we do not take into account all possible interferences of moving points, moving triangle faces and moving triangle sides but restrict ourselves to three examples.

The first collision of $p_{1}(t)$ and $Q$ where $p_{1}(t)$ lies inside $Q$ occurs at $t=0.8271$ (Figure 2, top right). Collisions between the moving triangle $P$ and vertices of $Q$ are outside the parameter interval $[0,1]$. For example, the collisions of $q_{1}$ and $P$ are at $t=-0.1917$ and $t=1.5461$. Finally, the intersection of two lines $p_{1} \vee p_{2}$ and $q_{0} \vee q_{1}$ occures at $t=0.2568$ and $t=1.4692$. The first collision is also the first relevant collision between the triangles $P$ and $Q$, see Figure 2, bottom right.

\section{Conclusion}

We showed how to use biarcs on the Study quadric for the construction of smooth Hermite interpolating Bennett motions and we proposed two choices of the joint position that guarantee bi-invariance. We also discussed how to perform basic collision tests between moving and static objects with polyhedral boundaries. The application of locally approximating Bennett motions and of Bennett motions in the simulation of interacting particle systems, where approximate collisions need to be estimated from position, velocity and acceleration data, seem to be promising topics of further research.

Acknowledgements The second author has been supported by the Austrian Science Fund through the National Research Network S92 "Industrial Geometry", subproject 02. The authors thank M. Rabl for many useful discussions on biarcs. 

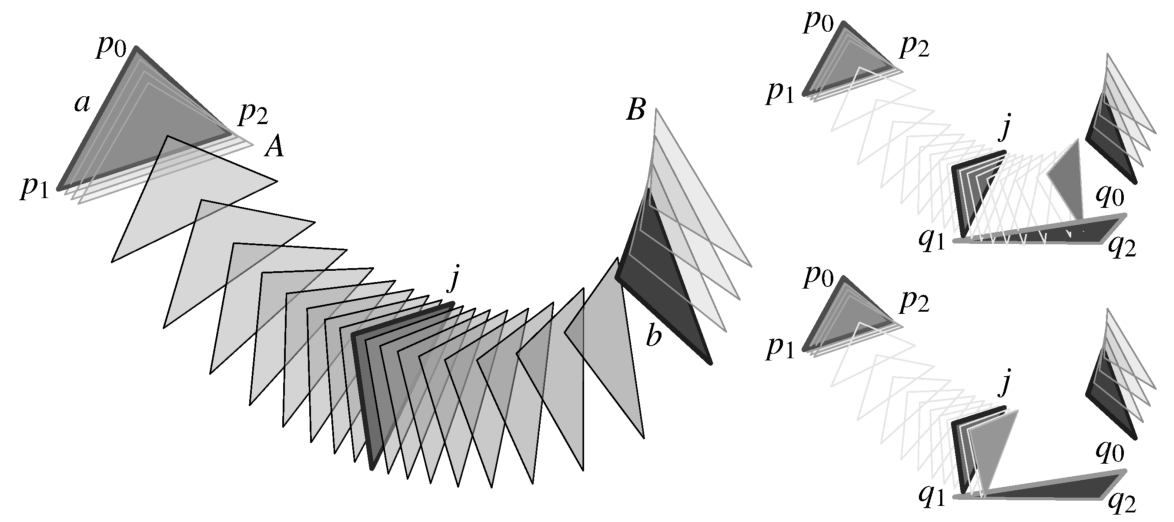

Fig. 2 Biarc data and resulting Bennett motion (left); collisions with a fixed triangle: moving point vs. fixed plane (top right) and moving line vs. fixed line (bottom right).

\section{References}

1. Baker, J.E., On the motion geometry of the Bennett linkage. In Proc. 8th Int. Conf. on Engineering, Computer Graphics and Descriptive Geometry, 433-437, Austin TX (1998)

2. Bennett, G.T., A new mechanism. Engineering 76, 777-778 (1903)

3. Bennett, G.T., The skew isogramm-mechanism. Proc. London Math. Soc. (2nd series) 13, 151-173 (1913-1914)

4. Bottema, O. and Roth, B., Theoretical Kinematics. Dover (1990)

5. Brunnthaler, K., Synthesis of 4R Linkages Using Kinematic Mapping. Ph.D. thesis, University Innsbruck (2007)

6. Brunnthaler, K., Schröcker, H.-P., and Husty, M., A new method for the synthesis of Bennett mechanisms. In Proc. Int. Workshop on Computational Kinematics, Cassino (2005)

7. Fuhs, W. and Stachel, H., Circular pipe-connections. Comput. Graphics 12(1), 53-57 (1988)

8. Husty, M. and Schröcker, H.-P., Algebraic geometry and kinematics. In I. Emiris, F. Sottile, and T. Theobald (eds.) Nonlinear Computational Geometry, IMA (accepted for publication)

9. Jung, S., Hong, M., and Choi, M.-H., An adaptive collision detection and resolution for deformable objects using spherical implicit surface. In V. Sunderam et al. (eds.) ICCS 2005, volume 3514 of $L N C S$, 735-742, Springer (2005)

10. Jüttler, B. and Wagner, M.-G., Kinematics and animation. In G. Farin, J. Hoschek, and M.S. Kim (eds.) Handbook of Computer Aided Geometric Design, chapter 29, 723-748, Elsevier, Amsterdam (2002)

11. McCarthy, J.M., An Introduction to Theoretical Kinematics. MIT Press, Cambridge, Massachusetts, London, England (1990)

12. Peternell, M., $G^{1}$-Hermite interpolation of ruled surfaces. In T. Lyche and L.L. Schumaker (eds.) Mathematical Methods in CAGD: Oslo 2000, 413-422, Vanderbilt Univ. Press., Nashville (2001)

13. Pottmann, H. and Wallner, J., Computational Line Geometry. Mathematics and visualization, Springer, Heidelberg (2001)

14. Röschel, O., Rational motion design - a survey. Comput. Aided Design 30(3), 169-178 (1998)

15. Wang, W. and Joe, B., Orientation interpolation in quaternion space using spherical biarcs. In Proc. Graphics Interface '93, 24-32 (1993)

16. Wang, W. and Joe, B., Interpolation on quadric surfaces with rational quadratic spline curves. Comput. Aided Geom. Design 14(3), 207-230 (1997)

17. Weiss, E.A., Einführung in die Liniengeometrie und Kinematik. Teubners Mathematische Leitfäden, B. G. Teubner, Leipzig, Berlin (1935) 